\title{
Indirect Acoustic Impedance Eduction in Presence of Flow Based on an Analytical Two-Port Formulation
}

\author{
Leandro D. Santana ${ }^{\mathrm{a}}$, Wim De Roeck ${ }^{\mathrm{a}}$, Wim Desmet ${ }^{\mathrm{a}}$ \\ ${ }^{a}$ K.U. Leuven, Department of Mechanical Engineering, Celestijnenlaan 300B, B-3001 \\ Heverlee (Leuven), Belgium \\ Email: Leandro.DeSantanaDantas@mech.kuleuven.be \\ Phone: +32 16 322672 \\ Fax: +32 16322987
}

\begin{abstract}
In order to assess the performance of advanced acoustic absorbing materials the acoustic impedance should be determined with high accuracy, especially, in presence of a grazing flow. This paper presents an analytical methodology for the grazing flow acoustic impedance eduction, based on a two-port representation of an acoustic system. This procedure is dedicated to the frequency range below the cut-on frequency of the transversal duct modes and its major appeal refers to a reduced number of microphones and the experimental rig simplicity. The methodology is verified by comparison with acoustic finite element simulations. Experiments are performed on two liner configurations and the educed impedance is compared with reference results obtained with a different approach at another test rig, and shows good agreement. A sensitivity analysis on the influence of the input parameters is carried out showing the influence of the rigid-/impedance wall transition effects, flow Mach number, number of microphones and microphone positioning accuracy.
\end{abstract}

Keywords: aeroacoustics, acoustic liner, acoustic impedance, grazing flow indirect impedance eduction, two-port formulation

\section{Introduction}

Acoustic absorbing materials are commonly used in many applications, ranging from the automotive industry to the civil constructions. It is, however, in the aeronautical sector that they find the most challenging requirements of robustness, low weight and high performance. In commercial air- 
planes, the aircraft engine is one of the main noise sources, producing noise levels which can exceed $160 \mathrm{~dB}$. In view of a sustainable and environment friendly civil aviation, engine noise reduction through efficient noise mitigation is required. One of the most common solutions to reduce the far-field engine noise is to line the engine nacelle. The acoustic liner is constructed by a rigid backing plate, covered by a honeycomb structure, which, in turn, is covered by a perforated plate. This construction behaves like a Helmholtz resonator, but, due the covering perforated plate, construction imperfections and the presence of grazing flows, the acoustic characteristics of these materials change drastically. Direct and indirect impedance measurement techniques have been used to characterize acoustic absorbing materials, but with the development of new high performance and multiple degrees of freedom acoustic liners, improved techniques are required to accurately determine the acoustic impedance including all physical complexities.

The direct methods are still commonly used in engineering and scientific applications. The most basic and commonly used direct methods to determine the acoustic impedance, are the two microphone technique and the insitu method $[1,2]$. This last methodology, specifically designed for acoustic liners, assumes that for a resonant cavity a unique relationship exists between the internal acoustic pressure and the acoustic particle velocity along the perforated plate. This technique is valid under the hypothesis of long wavelengths compared to the cavity cross dimensions and the assumption that the locally measured liner properties are extendable to the full extent of the material surface. The main disadvantages of this methodology are the fact that it is intrusive to the sample, since the microphone is positioned inside the sample, and it requires drill holes in the sample for microphone positioning, which can change the system characteristics and may render the material useless after the experiment.

Opposite to the direct techniques, indirect methods emerged as an alternative to avoid the restrictions of these methods and bring additional advantages. Among several indirect methods the Finite Element techniques have been adopted as a first, and are still relevant $[3,4,5,6,7]$. This technique consists of solving an eigenvalue problem, where the material impedance is treated as an unknown and the measured acoustic field corresponds to the boundary condition. This technique successfully allows integrating flow velocity gradients along any dimension of the testing section and can cope with materials with acoustic impedance variation along any dimension.

Mode-Matching Techniques $[8,9,10]$ are a promising alternative for the 
acoustic impedance eduction. The Mode-Matching Technique is based on a modal decomposition of the acoustic pressure and velocity fields, which can be represented as a set of algebraic equations. This technique allows the computation of characteristic acoustic pressure and velocities of high order modes, with a cost of requiring a large number of microphones. In this way, it is possible to accurately determine the acoustic impedance at small wave numbers compared to the duct dimensions and to directly take into account the rigid/impedance wall transition effects unavoidably present in every test rig [10].

As a trade-off between the Mode-Matching Techniques complexity and good accuracy, two-port methodologies have been presented [11, 12]. This alternative permits to adopt a less complex rig and produces results with comparable accuracy to the Mode-Matching Techniques. The two-port technique computes the upstream and the downstream propagating acoustic waves on each side of the duct, where the acoustic sample is located. Supposing acoustic plane wave propagation, it is possible to obtain a unique relationship between the upstream and downstream propagating waves and the acoustic impedance. This technique benefits from the reduced number of microphones required to accurately compute the acoustic impedance, it does not require an anechoic termination and, with the use of a polyharmonic distortion model [13], it is possible to take into account non-linear effects in the two-port transfer matrix.

The present paper studies an analytical indirect method for the acoustic impedance eduction based on a two-port formulation. Under the assumption that acoustic plane wave propagation is occurring, this technique enables the acoustic impedance eduction, under grazing flow conditions, using a minimum of four microphones. Furthermore, the methodology is able to determine the rigid-/impedance-wall transition effects leading to a more accurate acoustic impedance prediction. The adopted technique is theoretically presented and verified by comparison with acoustic finite element simulations. Afterwards, experiments are conducted on two acoustic liner samples which are representative for commercial aircraft nacelles. The educed impedance of these samples is compared with reference values, obtained using a different methodology, on a different test rig, and are found to be in good agreement. Finally, a sensitivity analysis discusses the influence of the input parameters and physical effects which should be taken into account to obtain an accurate impedance eduction using the two-port methodology presented in this paper. 


\section{Acoustic two-port systems}

\subsection{The linear acoustic network}

A two-port network [14] is defined as an acoustic system located in between two straight ducts where the acoustic plane wave propagation hypothesis is valid. A two-port system is fully characterized by an acoustic transfer matrix $(T)$ which can be written as a relation between the acoustic pressure and velocity fluctuations on both sides of the two-port system. Among the different approaches to write the transfer matrix, this paper adopts two formulations: one which relates the right- and left-running acoustic waves $\left(p^{+}\right.$, $p^{-}$), on both sides of the system, the so-called scatter matrix $T_{s}$ (Eq. 1), and the other that relates the acoustic pressure and velocities $(p, u)$ on both sides of the two-port systems, also known as the acoustic transfer matrix $T$ (Eq. 2).

$$
\begin{gathered}
\left\{\begin{array}{c}
p_{2}^{+} \\
p_{1}^{-}
\end{array}\right\}=\left[T_{s}\right]\left\{\begin{array}{c}
p_{1}^{+} \\
p_{2}^{-}
\end{array}\right\}=\left[\begin{array}{ll}
T^{+} & R^{-} \\
R^{+} & T^{-}
\end{array}\right]\left\{\begin{array}{c}
p_{1}^{+} \\
p_{2}^{-}
\end{array}\right\} \\
\left\{\begin{array}{c}
p(l) \\
u_{z}(l)
\end{array}\right\}=[T]\left\{\begin{array}{c}
p(0) \\
u_{z}(0)
\end{array}\right\}=\left[\begin{array}{ll}
T_{11} & T_{12} \\
T_{21} & T_{22}
\end{array}\right]\left\{\begin{array}{c}
p(0) \\
u_{z}(0)
\end{array}\right\}
\end{gathered}
$$

In Eq. 1, the indices 1 and 2 represent, respectively, the upstream and the downstream part of the two-port system. In Eq. 2, the indices 0 and $l$ respectively represent the plane, upstream and downstream, where the transition rigid-/impedance wall occurs. De Roeck [15] presents a formulation which uniquely relates Eqs. 1 and 2.

\subsection{Upstream and downstream propagating waves}

A minimum of two microphones are needed to define the complex upstream $\left(p^{+}\right)$and downstream $\left(p^{-}\right)$propagating waves. Assuming plane wave propagation, for a fixed reference plane $z=0$, the right- and the left-running acoustic pressure field at an arbitrary position $z$ is directly related to the reference axial position $z=0$ by the relationship:

$$
p^{\prime}(z, f)=p^{+}(z, f)+p^{-}(z, f)=p^{+}(0, f) e^{-j k^{+} z}+p^{-}(0, f) e^{j k^{-} z}
$$

where the right- and the left-running acoustic wave numbers are defined, respectively, as $k^{+} \approx k_{0} /(1-M)$ and $k^{-} \approx k_{0} /(1+M)$ [2]. The Mach number $M$ is defined as the ratio of the flow velocity $\left(U_{0}\right)$ to the sound speed $\left(c_{0}\right) M=U_{0} / c_{0}$. The wavenumber $k_{0}$ is defined as $k_{0}=\omega / c_{0}$, with 
$\omega=2 \pi f$, where $f$ is the frequency. An additional correction of the upstream and downstream wave number, based on a quasi 2D approach, can be used for non space-uniform mean flow conditions [16]. Equation 3 can be further generalized on each side of the two-port system, for an arbitrary number of $n$ microphones, as described in Eq. 4:

$$
\left\{\begin{array}{l}
p^{+}(f) \\
p^{-}(f)
\end{array}\right\}=\left[\begin{array}{cc}
e^{-j k^{+} z_{1}} & e^{j k^{-} z_{1}} \\
e^{-j k^{+} z_{2}} & e^{j k^{-} z_{2}} \\
\vdots & \vdots \\
e^{-j k^{+} z_{n}} & e^{j k^{-} z_{n}}
\end{array}\right]^{\otimes}\left\{\begin{array}{c}
p^{\prime}\left(z_{1}, f\right) \\
p^{\prime}\left(z_{2}, f\right) \\
\vdots \\
p^{\prime}\left(z_{n}, f\right)
\end{array}\right\}
$$

where $\otimes$ represents the Moore-Penrose pseudo matrix inverse operator and $z_{k}$ the location of the $k^{t h}$ microphone relative to the reference plane.

The overdetermined formulation of Eq. 4 is advantageous, since the least squares solution of this system of equations, based on Moore-Penrose pseudo matrix inverse technique, suppresses random errors, such as those generated by aerodynamic pressure fluctuations in the ducts, noise on the acquisition system and small measurement errors. As a consequence, it can significantly increase the signal-to-noise ratio.

In addition, effects of fully developed boundary layers can be considered on the computation of $p^{+}$and $p^{-}$, on according to Dokumaci [17], with the addition of propagation constants $K^{+}$and $K^{-}$:

$$
p^{+}(x)=e^{i k K^{+} x} p^{+}(0), \quad p^{-}(x)=e^{i k K^{-} x} p^{-}(0)
$$

these correction factors are dependent of the flow Mach number, section geometry and the turbulent boundary layer profile.

\subsection{Two-port matrix coefficients}

At least two measurements are needed to determine the scatter matrix coefficients of Eq. 1. There are three approaches to obtain the two-port coefficients: the first consists of varying the position of the external sound source (two-source technique) [14, 18]; the second adopts a different impedance termination for each experiment (two-load technique) [19]; and the third adopts a combination of these two techniques. The latter approach is used in this 
paper. The two-port Eq. 1 can be written for $m$ experiments as:

$$
\begin{aligned}
& {\left[\begin{array}{ll}
T^{+}(f) & R^{-}(f) \\
R^{+}(f) & T^{-}(f)
\end{array}\right]=} \\
& {\left[\begin{array}{llll}
p_{2,1}^{+}(f) & p_{2,2}^{+}(f) & \cdots & p_{2, m}^{+}(f) \\
p_{1,1}^{-}(f) & p_{1,2}^{-}(f) & \cdots & p_{1, m}^{-}(f)
\end{array}\right]\left[\begin{array}{llll}
p_{1,1}^{+}(f) & p_{1,2}^{+}(f) & \cdots & p_{1, m}^{+}(f) \\
p_{2,1}^{-}(f) & p_{2,2}^{-}(f) & \cdots & p_{2, m}^{-}(f)
\end{array}\right]^{\otimes} .}
\end{aligned}
$$

Similar as for the determination of the left- and right-running waves, a least squares strategy can be used to solve Eq. 6, to further improve the signal-tonoise ratio. As a result, an overdetermined system of $m$ equations and four unknowns: $T^{+}, T^{-}, R^{+}$and $R^{-}$is obtained.

\section{Indirect impedance determination with flow}

\subsection{Problem description}

A straight duct with rectangular cross section $b \times h$ and length $l$, shown in Fig. 1, is considered. Inside the duct a uniform mean flow in the $z$-direction with a Mach number $M$ is considered. All walls are assumed to be perfectly rigid, except for the lined wall, localized at the upper $x$-boundary $(x=b)$. Outside the lined section, the hypothesis of acoustic plane wave propagation is assumed to be valid up to a maximum frequency $f<c_{0} \beta /(2 h)$, where $\beta=\sqrt{1-M^{2}}$, corresponding to the first transversal resonance frequency of the duct. As a result, the frequency range can be further extended by reducing the cross section of the duct up to a limit where the contribution from the edges at the lower and upper part of the impedance sections affects the estimation of the actual acoustic impedance under grazing flow.

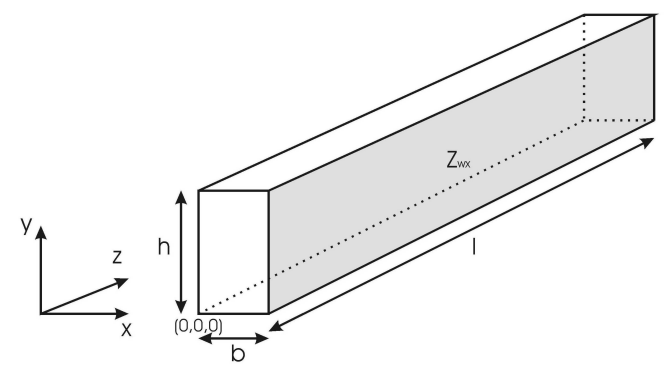

Figure 1: Rectangular duct with cross sectional dimensions $b \times h$ and length $l$ with impedance $Z_{w x}$ imposed on the boundary $x=b$. 


\subsection{Analytical two-port matrix formulation}

The pressure field inside the single lined duct can be written as a function of the right- and left-running acoustic waves, propagating with the respective wavenumbers $k_{z}^{+}$and $k_{z}^{-}$:

$$
\begin{aligned}
p(x, y, z, t) & =p^{+}(x, y, z, t)+p^{-}(x, y, z, t) \\
& =\left(A(x, y) e^{-j k_{z}^{+} z}+B(x, y) e^{j k_{z}^{-} z}\right) e^{j \omega t}
\end{aligned}
$$

where $A(x, y)$ and $B(x, y)$ represent the amplitude and the cross-sectional variation of the pressure fluctuations for, respectively, the right- and leftrunning acoustic waves. The linearized momentum equation reads as:

$$
\rho_{0} \frac{D u_{z}}{D t}+\frac{\partial p}{\partial z}=\rho_{0} \frac{\partial u_{z}}{\partial t}+Z_{0} M \frac{\partial u_{z}}{\partial z}+\frac{\partial p}{\partial z}=0
$$

with $\rho_{0}$ the mean density, $Z_{0}=\rho_{0} c_{0}$ the air characteristic acoustic impedance and $M=U_{0} / c_{0}$ the mean flow Mach number in the lined section. The axial velocity fluctuation $u_{z}$ for the right- and left-running acoustic waves are defined as:

$$
\begin{aligned}
u_{z}(x, y, z, t) & =u_{z}^{+}(x, y, z, t)+u_{z}^{-}(x, y, z, t) \\
& =\left(\frac{1}{Z^{+}} A(x, y) e^{-j k_{z}^{+} z}-\frac{1}{Z^{-}} B(x, y) e^{j k_{z}^{-} z}\right) e^{j \omega t}
\end{aligned}
$$

where $Z^{+}$and $Z^{-}$are defined as:

$$
Z^{+}=Z_{0}\left(\frac{k_{0}-M k_{z}^{+}}{k_{z}^{+}}\right) \quad Z^{-}=Z_{0}\left(\frac{k_{0}+M k_{z}^{-}}{k_{z}^{-}}\right)
$$

Eliminating the propagation constants $A(x, y)$ and $B(x, y)$, and relating the acoustic pressure and velocity at the upstream $(z=0)$ and downstream $(z=l)$ end of the duct, leads to the following transfer matrix formulation:

$$
\left\{\begin{array}{c}
p(l) \\
u_{z}(l)
\end{array}\right\}=\left[\begin{array}{cc}
\frac{Z^{+} e^{-j k_{z}^{+} l}+Z^{-} e^{j k_{z}^{-} l}}{Z^{+}+Z^{-}} & \frac{Z^{+} Z^{-}\left(e^{-j k_{z}^{+} l}-e^{j k \bar{z}} l\right)}{Z^{+}+Z^{-}} \\
\frac{e^{-j k_{z}^{+} l}-e^{j k \bar{z} l}}{Z^{+}+Z^{-}} & \frac{Z^{-} e^{-j k_{z}^{+} l}+Z^{+} e^{j k_{z}^{-} l}}{Z^{+}+Z^{-}}
\end{array}\right]\left\{\begin{array}{c}
p(0) \\
u_{z}(0)
\end{array}\right\}
$$

Equation 11 can be compared with the experimentally or numerically obtained transfer matrix coefficients to determine the wavenumbers $k_{z}^{+}$and $k_{z}^{-}$. This comparison leads to an overdetermined system with 4 equations and 2 unknowns which can be solved using a least square methodology. 


\subsection{Boundary conditions}

The unknown impedance $Z_{w x}$ is incorporated in the wavenumbers $k_{z}^{+}$and $k_{z}^{-}$of Eq. 11. This is shown by writing the cross-sectional variation of the three-dimensional acoustic pressure field of the right- and left-running waves inside the duct:

$$
\begin{aligned}
& p^{+}(x, y, z, t)=C_{z}^{+} e^{-j k_{z}^{+} z}\left(e^{-j k_{x}^{+} x}+C_{x}^{+} e^{j k_{x}^{+} x}\right)\left(e^{-j k_{y}^{+} y}+C_{y}^{+} e^{j k_{y}^{+} y}\right) e^{j \omega \neq}(2) \\
& p^{-}(x, y, z, t)=C_{z}^{-} e^{j k_{z}^{-} z}\left(e^{-j k_{x}^{-} x}+C_{x}^{-} e^{j k_{x}^{-} x}\right)\left(e^{-j k_{y}^{-} y}+C_{y}^{-} e^{j k_{y}^{-} y}\right) e^{j \omega}(13)
\end{aligned}
$$

where $C_{z}^{+}, C_{z}^{-}, C_{x}^{+}, C_{x}^{-}, C_{y}^{+}$and $C_{y}^{-}$are propagation constants for the upstream and downstream propagating waves. For each cross-sectional mode $(m, n)$ in, respectively, the $x$ and $y$ direction, the compatibility relationship gives a relation between wavenumbers in the $x-, y-$ and $z$-direction:

$$
\begin{aligned}
k_{z, m, n}^{+2}+k_{x, m}^{+2}+k_{y, n}^{+2} & =\left(k_{0}-M k_{z, m, n}^{+}\right)^{2} \\
k_{z, m, n}^{-2}+k_{x, m}^{-2}+k_{y, n}^{-2} & =\left(k_{0}+M k_{z, m, n}^{-}\right)^{2}
\end{aligned}
$$

As the two-port methodology, adopted in this paper, relies on the hypothesis of plane acoustic wave propagation outside the lined section, only the least naturally attenuated mode $(m=n=0)$ is considered. For this reason the subscripts $m$ and $n$ are omitted in the remaining part of this paper.

Imposing the rigid wall boundary condition $(\partial p / \partial y=0)$ at $y=0$ and $y=h$ in Eq. 12, the wavenumber in the $y$-direction for the upstream- and downstream-running acoustic waves is obtained $\left(k_{y, n}^{+}=k_{y, n}^{-}=n \pi / h\right)$ and the $y$-dependency of the pressure field is expressed by an infinite summation of cosine functions:

$$
e^{-j k_{y}^{+} y}+C_{y}^{+} e^{j k_{y}^{+} y}=e^{-j k_{y}^{-} y}+C_{y}^{-} e^{j k_{y}^{-} y}=\sum_{n=0}^{\infty} \cos \frac{n \pi y}{h} .
$$

For the $(0,0)$ mode, it is thus possible to determine the wavenumbers in the $x$-direction $k_{x}^{+}$and $k_{x}^{-}$as:

$$
k_{x}^{+}=\sqrt{\left(k_{0}-M k_{z}^{+}\right)^{2}-k_{z}^{+2}} \quad k_{x}^{-}=\sqrt{\left(k_{0}+M k_{z}^{-}\right)^{2}-k_{z}^{-2}}
$$

Applying to the Eqs. 12 and 13 the right wall boundary condition $(\partial p / \partial x=$ 0 ) at $x=0$ results in $C_{x}^{+}=C_{x}^{-}=1$. Combining these equations with the 
linearized momentum equation (Eq. 8), in the $x$ - direction, the following relations are obtained for the acoustic velocity fluctuations in the upstream and downstream directions:

$$
\begin{aligned}
& u_{x}^{+}=\frac{C_{z}^{+}}{Z_{0}} \frac{k_{x}^{+}}{k_{0}} \frac{1}{\left(1-M \frac{k_{z}^{+}}{k_{0}}\right)}\left(e^{-j k_{x}^{+} x}-e^{j k_{x}^{+} x}\right) \sum_{n=0}^{\infty} \cos \frac{n \pi y}{h} e^{-j k_{z}^{+} z} e^{j \omega t} \\
& u_{x}^{-}=\frac{C_{z}^{-}}{Z_{0}} \frac{k_{x}^{-}}{k_{0}} \frac{1}{\left(1+M \frac{k_{z}^{-}}{k_{0}}\right)}\left(e^{-j k_{x}^{-} x}-e^{j k_{x}^{-} x}\right) \sum_{n=0}^{\infty} \cos \frac{n \pi y}{h} e^{j k_{z}^{-} z} e^{j \omega t}
\end{aligned}
$$

The impedance boundary condition at $x=b$ is based on the assumption that at this boundary the fluid particle displacement $\zeta$ and the wall particle displacement are identical. The acoustic impedance of the boundary and the velocity in the $x$-direction inside the duct are related to the particle displacement by:

$$
\frac{p}{Z_{w x}}=\frac{\partial \zeta}{\partial t} \quad u_{x}=\frac{D \zeta}{D t}
$$

The Ingard-Myers boundary condition formulation [20, 21] is obtained by eliminating the particle displacement at $x=b$, which results in:

$$
\frac{D p(b, y, z, t) / D t}{\partial u_{x}(b, y, z, t) / \partial t}=Z_{w x}
$$

Combining the Eqs. 12, 18 and 21 (or 13, 19 and 21) gives the solution for the unknown impedance at $x=b$ where $k_{z}^{+}$and $k_{z}^{-}$are obtained from the two-port determination and $k_{x}^{+}$and $k_{x}^{-}$from the compatibility relation 17 :

$$
Z_{w x}=j Z_{0} \frac{k_{0}}{k_{x}^{+}}\left(1-M \frac{k_{z}^{+}}{k_{0}}\right)^{2} \cot \left(k_{x}^{+} b\right)=j Z_{0} \frac{k_{0}}{k_{x}^{-}}\left(1+M \frac{k_{z}^{-}}{k_{0}}\right)^{2} \cot \left(k_{x}^{-} b\right)
$$

\subsection{The rigid-/impedance-wall transition}

The analytical formulation, discussed in the previous section, is valid for an infinitely long single wall lined duct, where no transition effects, due to the passage from the rigid to the lined wall, occur. In principle this assumption cannot be used for a two-port based eduction approach, since on the rigid/impedance wall transition region the plane wave propagation hypothesis is not fully valid. Adopting the schematic description of Fig. 2, an infinitesimal 
transition element is added on each side of the two-port system. No further hypothesis about the physical transition phenomena is made. This element is considered as a black-box with a transfer function able to represent linear effects. The acoustic pressure and velocities on both sides of the two-port system $\left(p_{A}, u_{A}, p_{D}, u_{D}\right)$ are related to the quantities on each side of the acoustic material sample $\left(p_{B}, u_{B}, p_{C}, u_{C}\right)$ by a transition matrix $T_{t r}$ and $T_{t r}^{-1}$, as a result, it is assumed that the hard wall-/impedance wall transmission properties, represented by $T_{t r}$, are identical at the upstream and downstream end of the lined section. This leads to the following relation between the acoustic pressure and velocity fluctuation at the different interfaces:

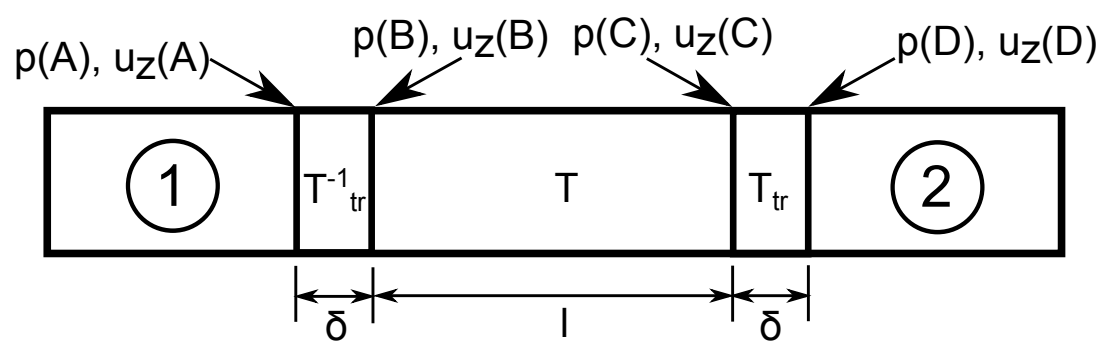

Figure 2: Impedance transition scheme. The sections (1) and (2) represent each side of the two-port system, the infinitesimal element, of length $\delta$ is represented by the transition matrix $T_{t r}$, and the unknown impedance element of length $l$ is represented by the matrix $T$.

$$
\begin{aligned}
\left\{\begin{array}{c}
p(l) \\
u_{z}(l)
\end{array}\right\}= & \left\{\begin{array}{c}
p(D) \\
u_{z}(D)
\end{array}\right\}=T_{t r}\left\{\begin{array}{c}
p(C) \\
u_{z}(C)
\end{array}\right\} \\
& \left\{\begin{array}{c}
p(C) \\
u_{z}(C)
\end{array}\right\}=T\left\{\begin{array}{c}
p(B) \\
u_{z}(B)
\end{array}\right\} \\
& \left\{\begin{array}{c}
p(B) \\
u_{z}(B)
\end{array}\right\}=T_{t r}^{-1}\left\{\begin{array}{c}
p(A) \\
u_{z}(A)
\end{array}\right\}=T_{t r}^{-1}\left\{\begin{array}{c}
p(0) \\
u_{z}(0)
\end{array}\right\}
\end{aligned}
$$

which can be simplified to the relationship:

$$
\left\{\begin{array}{c}
p(l) \\
u_{z}(l)
\end{array}\right\}=T_{\text {tot }}\left\{\begin{array}{c}
p(0) \\
u_{z}(0)
\end{array}\right\}=T_{t r} \times T \times T_{t r}^{-1}\left\{\begin{array}{c}
p(0) \\
u_{z}(0)
\end{array}\right\} .
$$

The $T_{t r}$ matrix coefficients should be deduced. No symmetric behavior is expected for the matrix $T_{t r}$, consequently, this matrix presents four unknowns, where at least two additional experiments/simulations are needed for the solution of the system of equations. 


\subsection{Impedance determination procedure}

For $m$ simulations/experiments, Eqs. 17, 22 and 24 result in a system with $2 * m+4$ equations and 9 unknowns $\left(k_{z}^{+}, k_{z}^{-}, k_{x}^{+}, k_{x}^{-}, Z_{w x}\right.$ and the four $T_{t r}$ matrix elements). For the no flow cases, the problem is simplified, since the respective right- and left-running wavenumbers are equal $\left(k_{z}^{+}=k_{z}^{-}\right.$and $k_{x}^{+}=k_{x}^{-}$), leading to a system with $2 * m+2$ equations and 7 unknowns. In both cases, the system of equations becomes overdetermined for a minimum of 3 simulations/experiments and can be solved using a least squares strategy.

The adopted algorithm for the impedance eduction, executes the following steps:

1. For each side of the two-port system, Eq. 4 is used to calculate the downstream and upstream propagating waves $\left(p^{+}\right.$and $\left.p^{-}\right)$, for each of the $m$ experiments. Equation 3 computes the associated total pressure $(p(0)$ and $p(l))$ and the Eq. 9 yields the velocity fluctuations $(u(0)$ and $u(l))$ at each end of the two-port system.

2. Equations 11, 17, 22 and 24 are adopted to form a system of $2 * m+4$ equations;

3. Given an expected impedance $Z_{w x}$, an initial guess for $k_{x}^{ \pm}$and $k_{z}^{ \pm}$is obtained using the Eq. 17 and 22, in addition, the matrix $T_{t r}$ is initialized by a unity matrix;

4. An optimizing procedure for the solution of a non-linear system of equations (i.e. the Matlab ${ }^{\circledR}$ fsolve routine) is adopted to solve the non-linear system of equations;

5. With $k_{z}^{ \pm}$determined in the previous step, relation 17 is used to obtain the $k_{x}^{ \pm}$and

6. Finally applying Eq. 22 yields the unknown impedance $Z_{w x}$.

\section{Numerical validation}

\subsection{Numerical model description}

A straight rectangular duct, with length $l$, width $b$ and height $h$ equal to, respectively: $1 \mathrm{~m}, 0.10 \mathrm{~m}$, and $0.05 \mathrm{~m}$, is chosen for the present validation. This geometry leads to a cut-on frequency of the transversal duct modes $(n=1)$ equal to $1700 \mathrm{~Hz}$. To include the rigid-/impedance wall transition effects, a rigid wall of $0.25 \mathrm{~m}$ length is added at the inlet and outlet of the tube. Consequently, the lined section has length $l$ of $0.5 \mathrm{~m}$ and is located in 
the central part of the duct, as shown in figure 3. A uniform structured hexahedral mesh is used. To ensure a minimal resolution of at least 10 points per wavelength, for the highest frequency of interest $(1700 \mathrm{~Hz})$, the size of each hexahedral element equals $5 \mathrm{~mm}$, which leads to an acoustic mesh containing 40000 elements. The acoustic finite element software LMS/Virtual.Lab ${ }^{\circledR}$ Rev

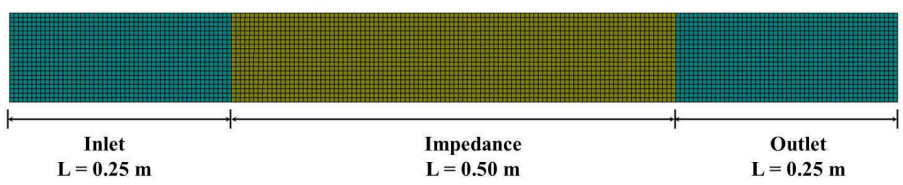

Figure 3: Representation of the finite element mesh and boundary conditions.

9.0 is used to calculate the acoustic field in the frequency domain. All walls are considered to be perfectly rigid, except in the region where the impedance is imposed. The inlet is excited using a vibrating panel. The outlet is defined as a wall with varying impedance values for each simulation, in order to obtain a variable loading [19]. On the duct wall where the impedance is applied, two different values are imposed: a constant impedance equal to $4 Z_{0}-4 i Z_{0}$ (with $i=\sqrt{-1}$ ) and a frequency-dependent impedance obtained using the Extended Helmholtz Resonator (EHR) Model [22]. The EHR input parameters are chosen in order to generate impedance values which trigger more pronounced hard-/soft wall transition effects. For both the frequencydependent and the frequency constant impedance simulations, a no-flow case $(\mathrm{M}=0)$ and a uniform mean flow case $(\mathrm{M}=0.3)$ are studied.

\subsection{Discussion of the results}

\subsubsection{No-flow cases}

The outcome of impedance eduction based on the finite element simulations results, for a quiescent medium, are shown in figures 4 and 5, for, respectively, the constant and frequency-dependent impedance cases. The left of figures 4 and 5, show the comparison between the imposed impedance in the finite element model and the one educed by the proposed methodology. The right of figures 4 and 5 present the relative error between the two impedances, defined as the modulus of the relative amplitude difference between the two values. 

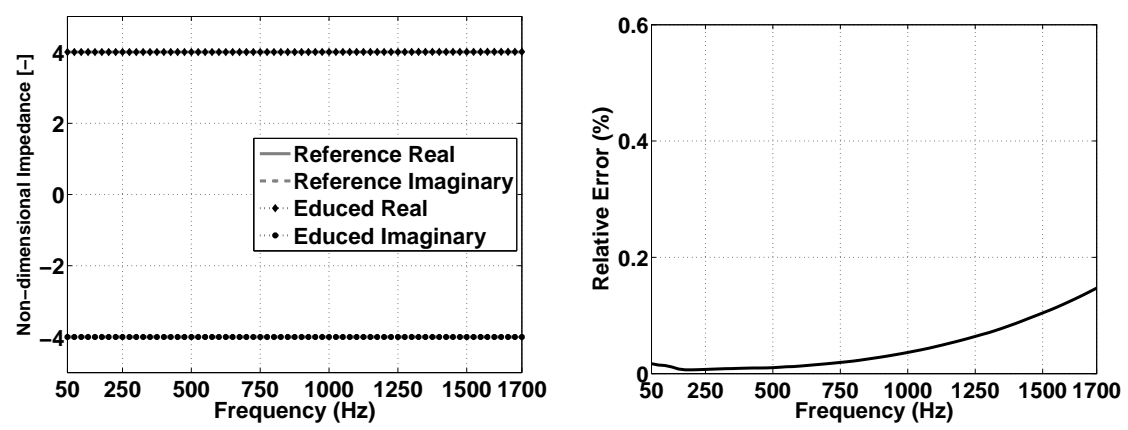

Figure 4: Comparison between the frequency-independent imposed and the educed impedance (left), and the relative error between these two impedances (right) $(\mathrm{M}=0)$.
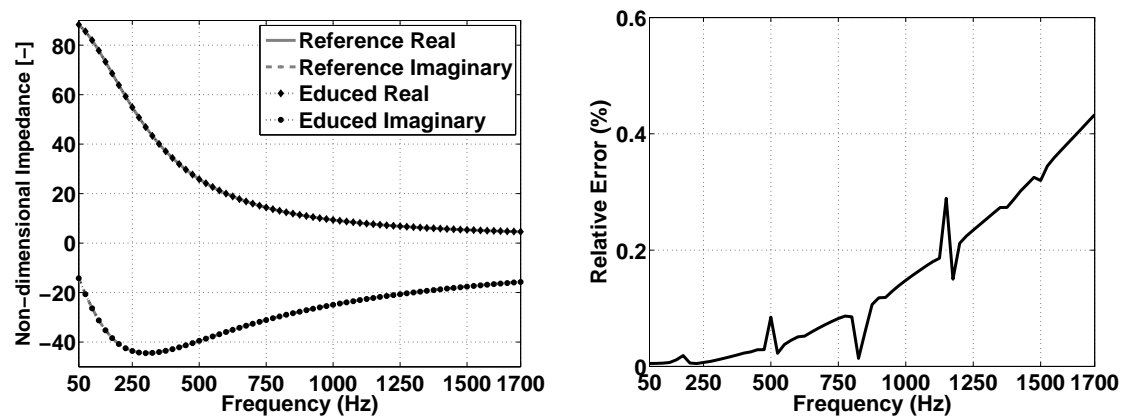

Figure 5: Comparison between the frequency-dependent imposed and the educed impedance (left), and the relative error between these two impedances (right) $(\mathrm{M}=0)$.

For both cases an excellent agreement between the imposed and the educed acoustic impedance is observed. The error grows following an exponential rule until the duct cut-on frequency. This behavior is, on the one hand, caused by the fact that the hypothesis of the acoustic plane wave propagation, on either side of the two-port system, becomes less valid at higher frequencies. The numerical accuracy, on the other hand, also decreases with higher frequencies due to dispersion errors. Some spikes are noticed on the error curve of figure 5, these originated from local instabilities in the solution procedure of the non-linear system of equations. As this problem is fully related to the difficulty of the optimization routine to find a solution within a given number of iterations, and affects only few points in the whole frequency spectrum, not jeopardizing the overall accuracy of the solution, no 
further attention is paid to this phenomenon.

\subsubsection{Flow cases}

In figures 6 and 7 the results for, respectively, the constant and frequencydependent impedance, are shown for a medium with a uniform mean flow with Mach number equal to 0.3. In these figures, the left part represents the comparison between the imposed and the educed impedance and the right part represents the relative error between these two values. The flow case
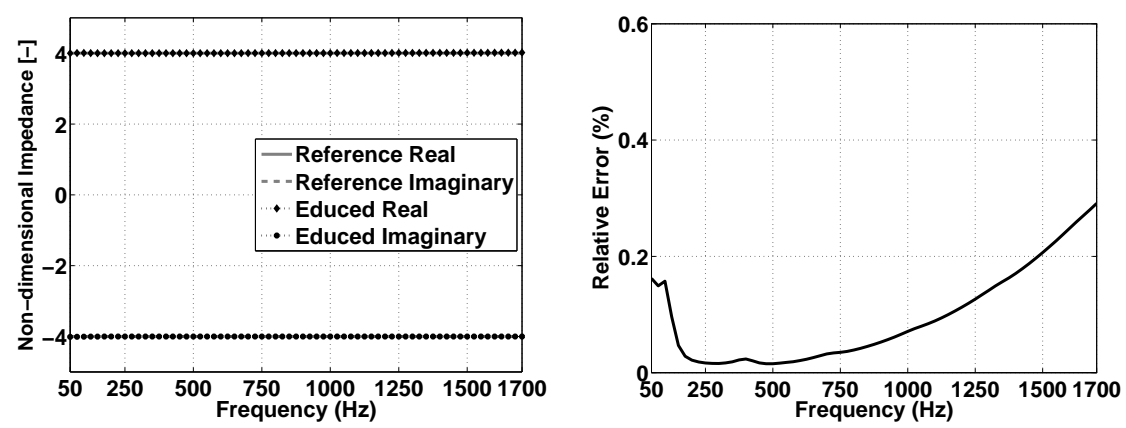

Figure 6: Comparison between the frequency-independent imposed and the educed impedance (left), and the relative error between these two impedances (right) $(M=0.3)$.
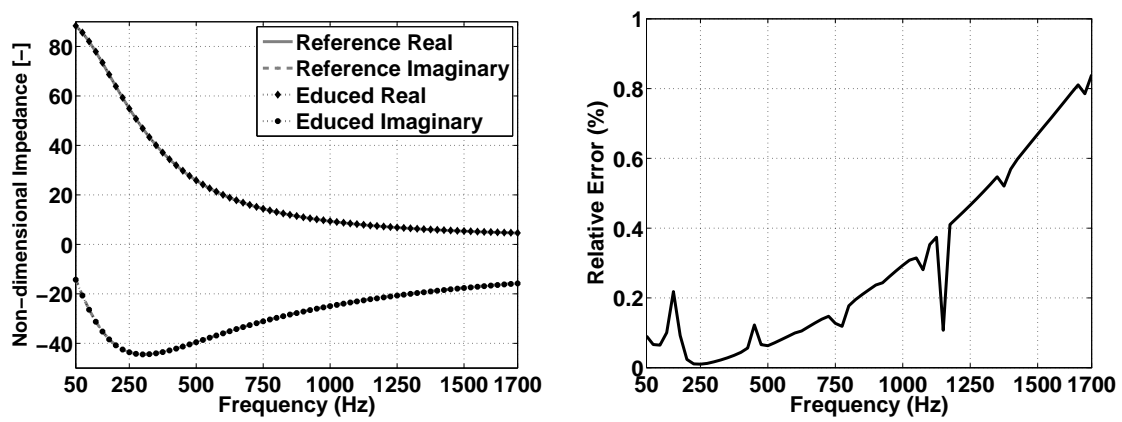

Figure 7: Comparison between the frequency-dependent imposed and the educed impedance (left), and the relative error between these two impedances (right) $(\mathrm{M}=0.3)$.

results presented in figures 6 and 7 also show an excellent agreement between the imposed and the educed impedance. The relative error, in amplitude, is slightly higher than the one for the no-flow cases. Similar as for the no-flow 
case, the error grows with increasing frequency, for both test cases, following an exponential pattern.

\section{Experimental set-up and results}

\subsection{The experimental facility}

An open circuit aeroacoustic test rig [23], is used for the experimental validation. The test facility allows an analysis of the aeroacoustic noise generation, absorption and propagation mechanisms for confined subsonic flow applications under various mean flow conditions. Figure 8 presents a schematic overview of the open flow circuit testing section.

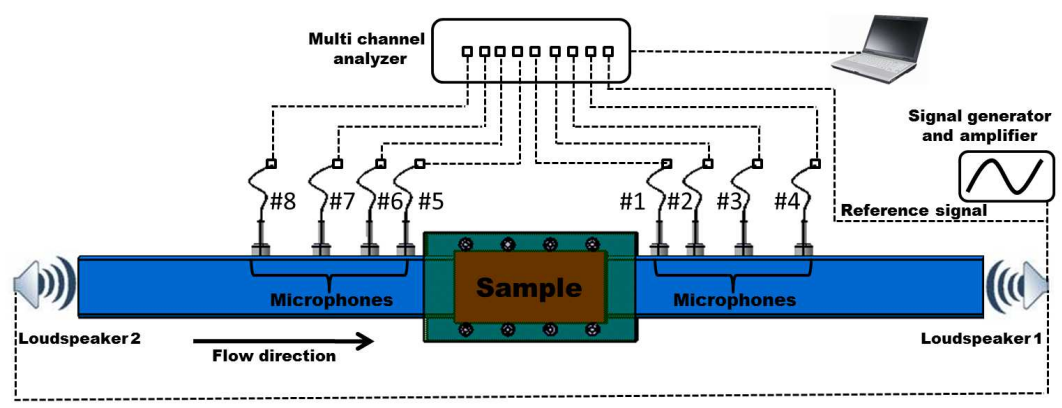

Figure 8: Schematic representation of the experimental rig.

Four microphones are used on each side of the two-port system with a sampling frequency of $25 \mathrm{kHz}$. As excitation signal a swept sine is used, which has a total duration of 20 seconds and a frequency range between $20 \mathrm{~Hz}$ to $3.5 \mathrm{kHz}$. The excitation signal is recorded and used as noise-free reference for reduction of uncorrelated noise, measured by the microphones, due to the flow [24]. For the no-flow cases, a total measurement time of 60 seconds is chosen, for the flow cases a total measurement time of 600 seconds is used. To ensure a good signal-to-noise relationship, the loudspeakers are adjusted to guarantee, at the sample position, a sound pressure level close to $120 \mathrm{~dB}$, over the whole frequency range. The internal duct cross-sectional dimensions are 40 by $90 \mathrm{~mm}$, with the acoustic sample placed along this last dimension. The mean Mach numbers under consideration are equal to 0, 0.1 and 0.17, where the latter corresponds to the maximum reachable flow speed for the test section geometry on the experimental flow facility. 
Table 1: Main geometric parameters of the analyzed liners samples, where SDOF abbreviates Single Degree of Freedom.

\begin{tabular}{lcc} 
Test object & Sample 1 & Sample 2 \\
\hline Identification: & Perforated Liner SDOF & Linear Liner SDOF \\
Type: & SDOF & SDOF \\
Cell depth: & $0.5 \mathrm{in}(12.7 \mathrm{~mm})$ & $1.21 \mathrm{in}(30.73 \mathrm{~mm})$ \\
Hole diameter: & 1.45 to $1.5 \mathrm{~mm}$ & $1.1 \mathrm{~mm}$ (wire mesh) \\
Hole placement: & Parallel & N/A \\
Porosity: & $\approx 4 \%$ & N/A \\
Open area ratio: & $\approx 4.3 \%$ & $\mathrm{~N} / \mathrm{A}$ \\
$\lambda / 4$ resonance: & $\approx 6752 \mathrm{~Hz}$ & $\approx 2790 \mathrm{~Hz}$ \\
Helmholtz resonance: & $\approx 1670 \mathrm{~Hz}$ & $\approx 623 \mathrm{~Hz}$
\end{tabular}

The assumption of uniform mean flow is adopted on the present work. This hypothesis is based on the fact that, for the present duct section, assuming a parabolic boundary turbulent boundary layer profile, it is expected a correction factor $K^{ \pm}$(ref. Eq. 5) of $0 \%, 2 \%$ and $4 \%$, for the mean flow Mach numbers of $0,0.1$ and 0.17 , respectively. This correction was verified to have negligible impact (less than $1 \%$ ) on the educed impedance values, for the frequency range of interest.

\subsection{Descriptions of the liner samples}

Two acoustic liner samples, previously studied by Busse et al. [11], are tested in this paper. In Busse et al. the acoustic impedance results are determined by comparing insertion loss measurements with a numerical optimization based on the Extended Helmholtz Resonator Model [22]. The same sample notation of Busse et al. is adopted in this paper and their results are denoted as the reference solution. The physical properties of these samples are presented in table 1 and the pictures of the samples are shown in Fig. 9. Sample 1 corresponds to an acoustic liner which is typically installed in narrow body aircrafts, this sample is of particular interest for aeronautical engineering applications. Sample number 2 is important to be analyzed because of its linear behavior, caused by the wire-mesh covering. 

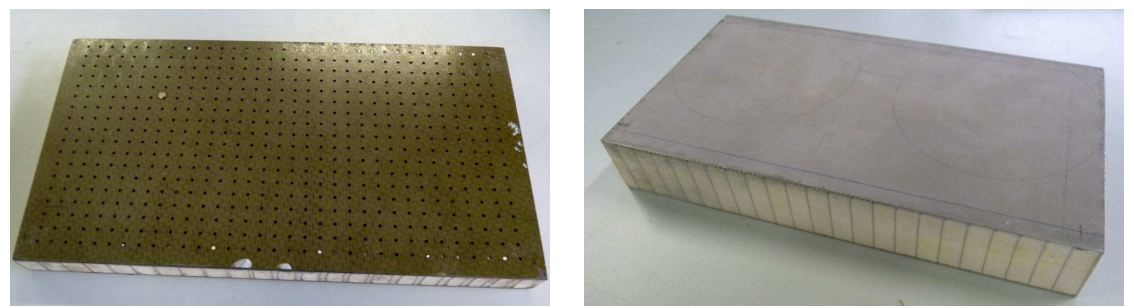

Figure 9: Test samples. Left: sample number 1 - perforated liner with single degree of freedom; Right: sample number 2 - linear liner with single degree of freedom.

\subsection{Discussion of the experimental results}

\subsubsection{Sample 1}

A comparison between the reference result and the educed impedance, using the technique described in this paper, is shown in Fig. 10. Figure 11 presents the relative amplitude difference between the reference and the educed results. From the Figs. 10 and 11 a good agreement is observed
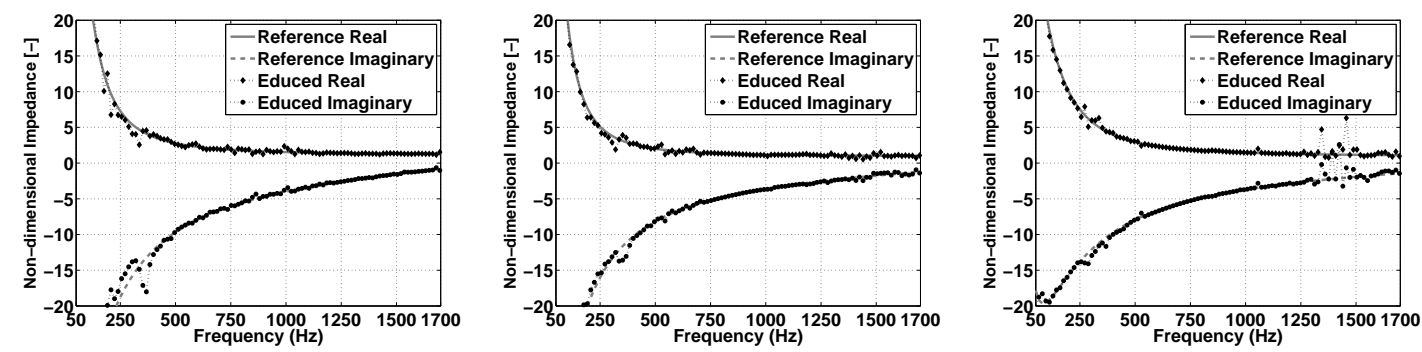

Figure 10: Comparison between the reference results and the impedance educed by the present technique for sample 1 . (Left $\mathrm{M}=0$, center $\mathrm{M}=0.1$ and right $\mathrm{M}=0.17$.)

between the reference solution and the educed one. The educed solution by this paper technique is less smooth than the reference one, mainly because the present technique does not adopt an impedance model, while the Busse et al. [11] results are based on an optimization of the coefficients for the Extended Helmholtz Resonator Model, imposing a specific frequency-dependent behavior. From the figures, it is noticed that at higher frequencies, additional oscillations occur which do not smooth out by increasing the sampling time (and as consequence the number of averages). It is observed that the presence of these oscillations becomes more pronounced at larger Mach numbers. This 

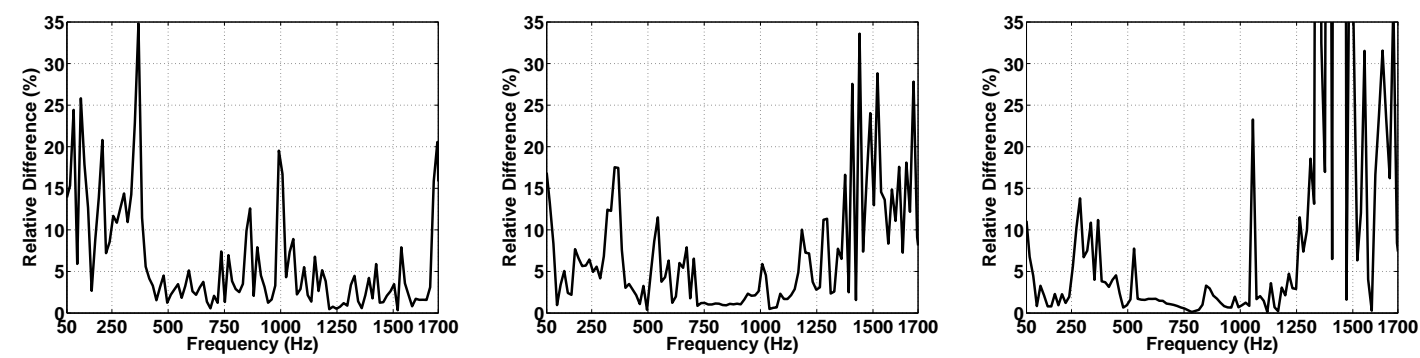

Figure 11: Relative amplitude difference between the reference impedance and the educed impedance by the present technique for sample 1 . (Left $M=0$, center $\mathrm{M}=0.1$ and right $\mathrm{M}=0.17$.)

indicates that, in the liner surface cavities, the flow is not linearly interacting with the acoustic field, as noticed by Marx et. al [25].

\subsubsection{Sample 2}

Figure 12 compares the educed impedance to the reference values for the sample 2, and Fig. 13 shows the relative amplitude difference for the same sample. A good agreement between the reference impedance values and
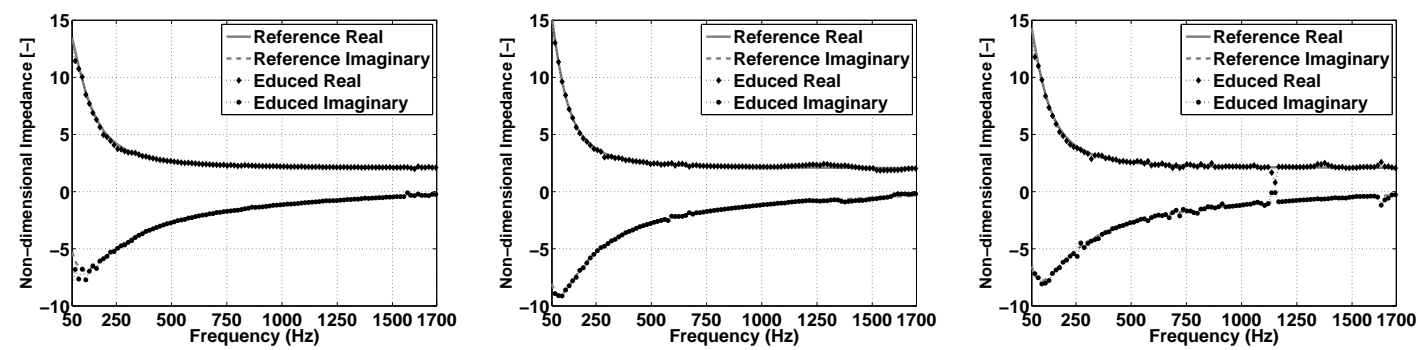

Figure 12: Comparison between the reference results and the impedance educed by the present technique for sample 2. (Left $\mathrm{M}=0$, center $\mathrm{M}=0.1$ and right $\mathrm{M}=0.17$.)

the educed results is observed. It is noticed that the oscillations on the educed impedance results are less pronounced, which is caused by the linear liner behavior due to the wire-mesh covering. Opposite to sample 1, there is less flow penetration in the liner cavities and consequently reduced nonlinear interactions between the acoustic and fluid dynamic field, at the liner surface. Observing the results at Mach 0.17 it is noticed that at frequencies 

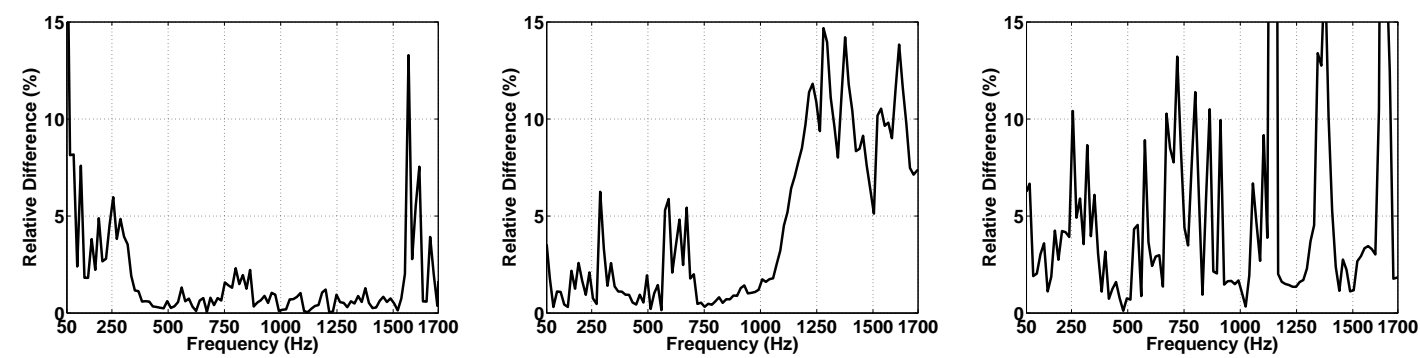

Figure 13: Relative amplitude difference between the reference impedance and the educed impedance by the present technique for sample 2. (Left $\mathrm{M}=0$, center $\mathrm{M}=0.1$ and right $\mathrm{M}=0.17$.)

close to 1100, 1300 and $1600 \mathrm{~Hz}$, a peak in the educed impedance solution is present. This corresponds to a single frequency where no solution was found by the optimization algorithm. Since this is only a local phenomenon given by the non-linear system of equations optimization algorithm, already noticed in the numerical simulations, this does not create a major problem to the validity of the methodology.

\section{Impedance eduction issues}

For an accurate impedance eduction, some parameters should be taken into account and carefully determined. In this section, the influence of different phenomena, i.e. the rigid-/impedance wall transition, the Mach number and microphone position determination and the proper initialization of the non-linear optimization algorithm is investigated.

\subsection{The rigid-/impedance-wall transition effects on the educed impedance}

One of the most significant influences on the impedance eduction results is the transition between the rigid and the impedance wall. Figure 14 presents, on the left side, the educed impedance, obtained experimentally on sample 2 at $M=0.1$, when the transition is taken into account and the right side shows the case where this effect is not considered. From Fig. 14, it is clear that the acoustic scattering, caused by the transition between the rigid and the impedance wall, plays an important role for all frequencies.

Similar transition effect are noticed for the finite element simulations. Figure 15 presents the effect of not taking into account the impedance transition in the impedance eduction methodology for the frequency dependent 

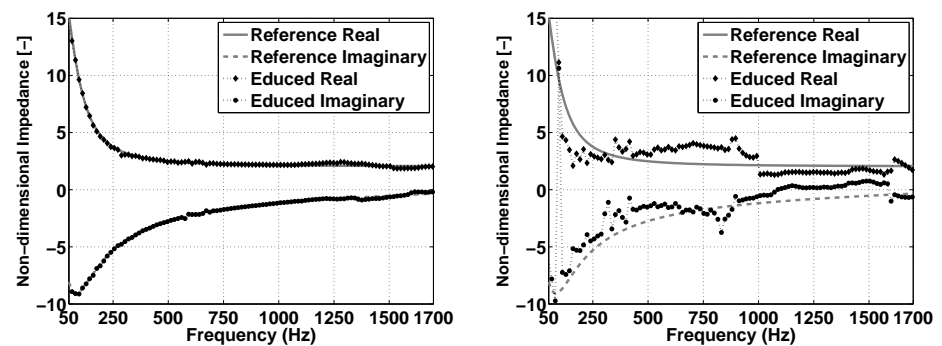

Figure 14: Rigid-/impedance wall transition effect on an experimental case. Reference result (left) with the rigid-/impedance wall effect taken into account, and without the impedance transition effect taken into account (right). Results for sample 2 and $\mathrm{M}=0.1$.

impedance at $M=0.3$. The left figure corresponds to the reference result, where the rigid-/impedance wall transition effect are taken into account, the right figure is the case where this effect is not considered. Figure 15 shows that oscillations on the educed impedance are caused by the sudden jump of impedance at the beginning and end of the liner sample [12]. From the figures it is clear that hard wall/soft wall transition effect can significantly influence the educed impedance results. An advantage of using a two-port approach to educe the impedance is the fact that the transition effect can be modeled using a black box representation. Thus, not requiring any additional assumptions about the physical phenomena which cause these transition effect.
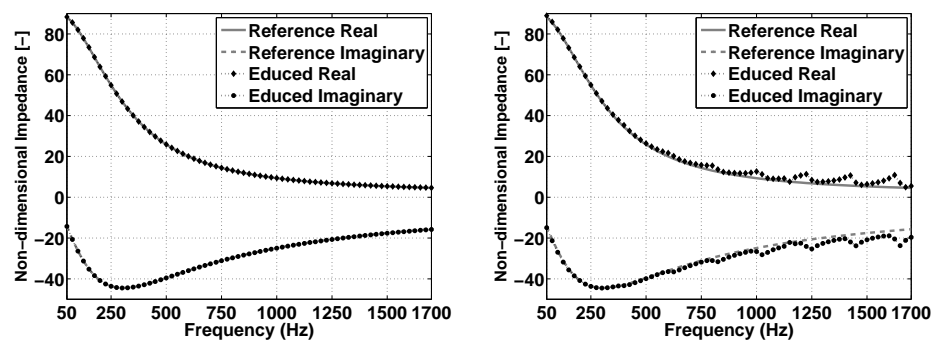

Figure 15: Rigid-/impedance wall transition effect for the numerical frequency-dependent impedance eduction. Reference result (left) with the rigid-/impedance wall effect taken into account, and without the impedance transition effect taken into account (right). Results for potential flow with $\mathrm{M}=0.3$. 


\subsection{The influence of the Mach number}

A proper estimation of the flow Mach number is an important parameter for an accurate impedance eduction. In a first approach, the duct flow Mach number is calculated using the velocity obtained by the mass flow measurements and the sound speed is estimated through the mean flow temperature. However, this approach was proven not to yield the required accuracy. For this reason a different approach is adopted, in which the 4 microphones on either side of the two-port system are used to compute the flow Mach number, the viscous losses and the effective upstream and downstream propagation wave number $k_{z}^{ \pm}[26]$. The Mach number, obtained at both sides of the twoport system is averaged over all frequencies and the final value is used for the impedance eduction calculations. To illustrate this effect, an error of $10 \%$ on the Mach number determination, is chosen as a realistic value. Figure 16 plots the educed impedance against the reference result when the nominal flow Mach number is 0.1. When comparing the results of Fig. 16, it is no-
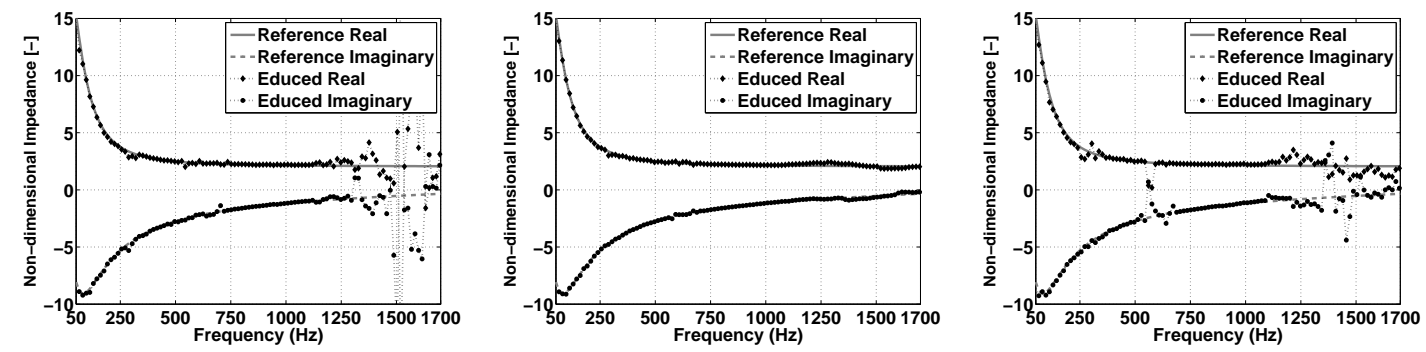

Figure 16: Impedance eduction for erroneous Mach numbers (left $M=0.09$, center $M=0.1$ and right $\mathrm{M}=0.11$ ).

ticed that an error of $10 \%$ on the Mach number determination, significantly influences the accuracy of the solution, mainly at higher frequencies. This fact also evidences the importance of avoiding flow speed fluctuations, during the data acquisition, for liner configurations that have a large sensitivity to the mean flow velocity.

\subsection{Influence of the microphone position}

The microphone position also plays an important role in the correct impedance eduction. Since it is difficult to determine the exact location of each microphone, an indirect distance measurement is preferred. Boonen 
Table 2: Microphone position $\left(z_{i}\right)$, uncertainty on the microphone positioning $(\delta)$ and fraction of the uncertainty $( \pm \% \delta)$ that each microphone was moved on each run of the impedance eduction routine.

\begin{tabular}{|c|c|c|c|c|c|}
\hline Mic. $\#$ & $z_{i}(\mathrm{~m})$ & $\delta(\mathrm{m})$ & $\pm \% \delta$ (left) & $\pm \% \delta$ (center) & $\pm \% \delta$ (right) \\
\hline 1 & -0.3350 & 0.0008 & 35.94 & -55.23 & -72.27 \\
\hline 2 & -0.4160 & 0.0012 & 31.01 & 50.25 & -70.14 \\
\hline 3 & -0.5480 & 0.0015 & -67.47 & -48.98 & -48.49 \\
\hline 4 & -0.7600 & 0.0020 & -76.20 & 1.19 & 68.14 \\
\hline 5 & 0.3330 & 0.0007 & -0.327 & 39.81 & -49.14 \\
\hline 6 & 0.4140 & 0.0014 & 91.94 & 78.18 & 62.85 \\
\hline 7 & 0.5450 & 0.0017 & -31.92 & 91.85 & -51.29 \\
\hline 8 & 0.7570 & 0.0020 & 17.05 & 9.44 & 85.85 \\
\hline
\end{tabular}

et. al [27] present a suitable technique that allows an accurate determination of the microphone position and quantification of the positioning error using a calibration measurement with a rigid termination. For the experimental test rig, used in this paper, the microphone positions and uncertainties are accurately calculated [28] and repeated on the $2^{\text {nd }}$ and $3^{\text {rd }}$ column of Tab 2 . In this table the microphones are numerated in such a way that, regarding Fig. 8, the microphones 1 to 4 and 5 to 8 are, respectively, localized downstream and upstream of the sample. The convention for microphone numeration considers that the microphone number grows from the sample position to the extremities of the two port system. The reference system origin was located in the geometric center of the duct.

To study the influence of the microphone positioning determination on the impedance eduction results, it is considered that each microphone is localized on a position $z_{i}$, where $i$ corresponds to the microphone number. For this analysis it is assumed that all microphone positions are moved from their most probable position $z_{i}$ by a random fraction of the uncertainty on the microphone positioning $\pm \delta$. The uncertainty on each microphone position is presented in the second column of Tab. 2. Figure 17 presents the results for three runs of the impedance eduction routine with microphones moved, by a random percentage, inside the uncertainty range $\pm \delta$. These percentage are, respectively, shown on the $4^{\text {th }}$ to the $6^{\text {th }}$ column of Tab 2.

Figure 17 shows, that small microphone positioning variations, even inside the uncertainty boundaries of the position measurement, cause significant 

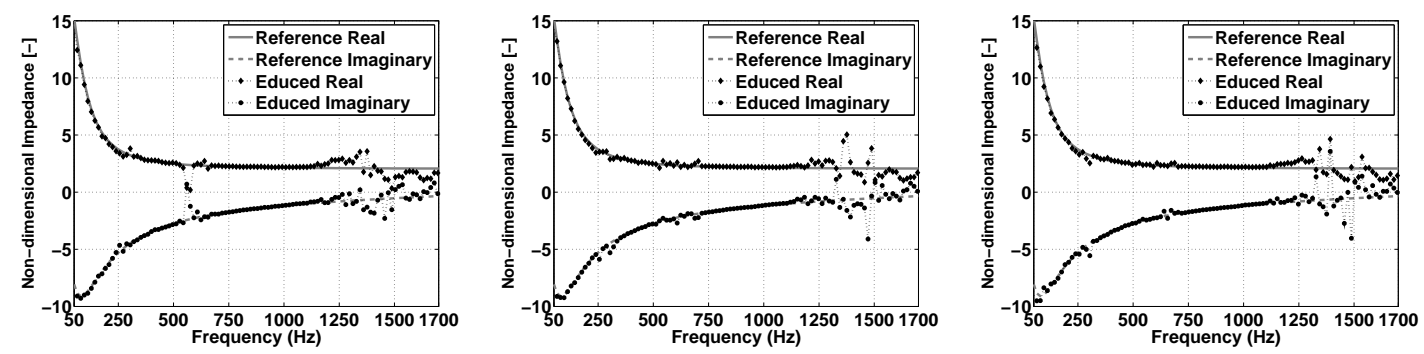

Figure 17: Impedance eduction for the microphone position displaced by a random fraction of its uncertainty. (Sample 2, M=0.1)

effect on the educed impedance, mainly at higher frequencies.

\subsection{The number of microphones effect}

Use the minimum number of microphones is important for reducing the experimental set-up cost. The present section discusses the main issue, noticed during the development of this work, related with the number of microphones. As demonstrated by Åbom and Bodén [24] the use of two microphones can cause errors of two distinct origin: the flow turbulence self-correlation between microphone pairs and the two microphones transferfunction singularity. The first is avoided when the distance between consecutive microphones is large enough to avoid, on the frequency range of interest, turbulence self-correlation, and, in this study, this issue has been verified of negligible effect. The second effect has been noticed as a major effect and is further discussed here.

Åbom and Bodén [24] demonstrates that the transfer function between two microphones has a minimum when the relationship $k \Delta z_{i}=n \pi\left(1-M^{2}\right)$ is satisfied, considering $n$ an integer and $\Delta z_{i}$ the spacing between microphone pairs. In addition, these authors suggests that the microphone spacing should be between the interval $0.1 \pi\left(1-M^{2}\right)<k \Delta z_{i}<0.8 \pi\left(1-M^{2}\right)$ in order to minimize the transfer-function related error. To evaluate these errors Fig. 18 plots the educed impedance for cases where two and three microphones are used on either side of the two-port system.

The left figure in Fig. 18 shows that the major disagreement between the reference and the educed results are present on the frequencies multiples of $400 \mathrm{~Hz}$, result which is in agreement with the relation $k \Delta z_{i}=1 \pi\left(1-M^{2}\right)$, considering the distance of $0.425 \mathrm{~m}$ between microphone pairs. The cen- 

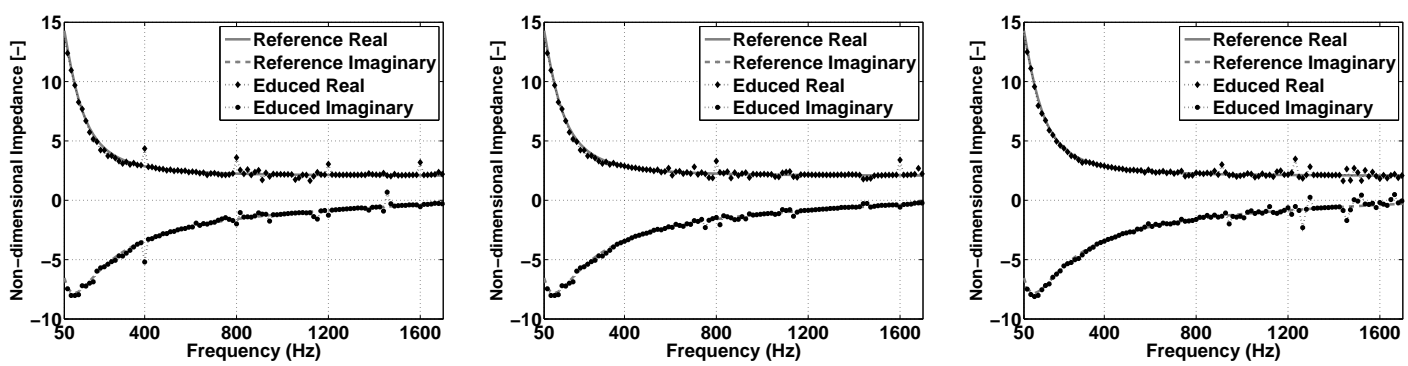

Figure 18: Number of microphones effect on the impedance. Left: impedance educed using microphones 1, 4, 5 and 8; Center: impedance educed using microphones 1, 2, 4, 5, 6 and 8; Right: impedance educed using microphones 1, 2, 5 and 6. (Sample 2, $\mathrm{M}=0.1$ )

tral figure in Fig. 18 shows that the addition of the microphones 2 and 5 between, respectively, the microphones 1 and 4,5 and 8 increases the frequency where the difference between expected and educed impedance mostly disagrees at frequencies multiple of $800 \mathrm{~Hz}$, value corresponding to the relationship $k \Delta z_{i}=2 \pi\left(1-M^{2}\right)$. Finally, the right figure in Fig. 18 allows to analyze that the relationship $0.1 \pi\left(1-M^{2}\right)<k \Delta z_{i}<0.8 \pi\left(1-M^{2}\right)$, which for the present case corresponds to the frequency range $65<f<527$, should, as well, be respected in order to avoid impedance eduction errors.

\subsection{The role of the optimization algorithm on the solution}

The last issue to be considered is the role of the optimization algorithm on the solution. For the present work the Matlab ${ }^{\circledR}$ Optimization Toolbox routine fsolve, with the Levenberg-Marquardt algorithm, is used to solve the non-linear system of equations. Figure 19 shows the educed impedance curve, where the initial guess, adopted as the reference solution, is varied by $-30 \%$ (left) and $+30 \%$ (right) with respect to the optimal initial guess. Figure 19 shows that, if the initial guess is not chosen in the vicinity of the actual solution, the function converges to a local minimum which does not correspond to the actual solution. To illustrate the convergence behavior of the optimization routine, the exit flag, for each point of the educed impedance curve, is presented in Fig. 20. The following list reproduces Matlab's manual for the exit flag meaning, indicating the convergence behavior of the root finding algorithm.

- -2 : Algorithm appears to be converging to a point that is not a root; 

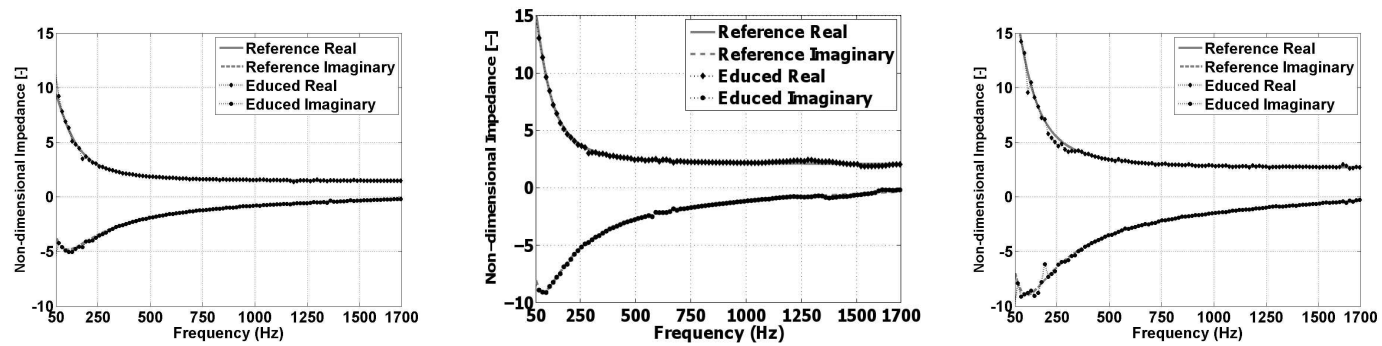

Figure 19: Educed impedance when the reference curve used as initial guess is subtracted with $30 \%$ (left), reference results (center) and educed impedance when the reference solution is increased with $30 \%$ (right). (Sample 2, M=0.0)
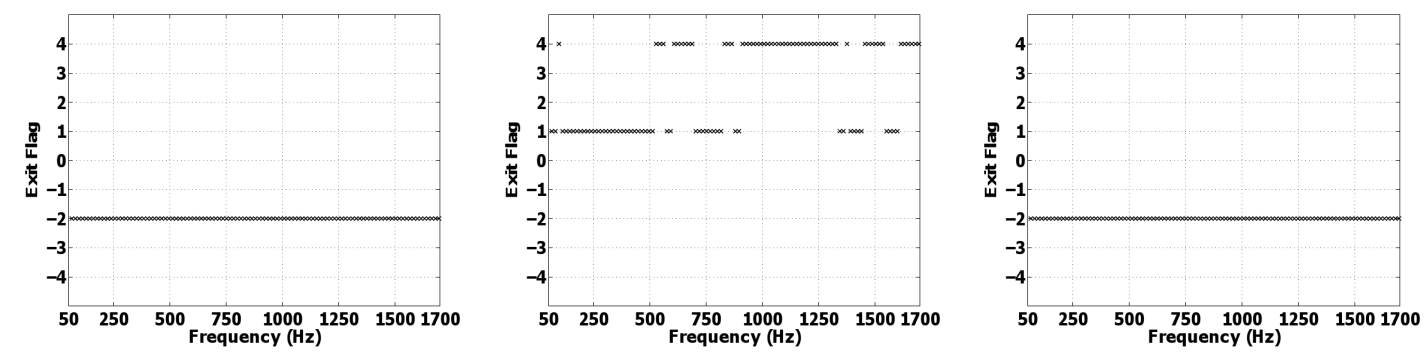

Figure 20: Exit flag when the reference values for the initial guess are subtracted with $30 \%$ (left), the exit flag for the reference results (center) and the exit flag when the reference results are increased with $30 \%$, in relation to the actual values. (Sample 2, M=0.0)

- 1: Function converged to a solution $\mathrm{x}$;

- 4: Magnitude of search direction was smaller than the specified tolerance.

Comparing the exit flags (Fig. 20) against its respective impedance curves (Fig. 19) and using the definition of the various exit flags, it is noticed that the left and right figures of Fig. 19 correspond to solutions where the algorithm converged to a local minimum. The central figure in Fig. 19 can be considered to be an actual solution due to the fact that the exit flag indicates that the algorithm converged to a point which is a solution, on some frequencies, and in others, that the magnitude of the search direction is smaller than the specified tolerance. This last exit flag should be carefully analyzed, even though the magnitude of the searching direction is smaller than the tolerance, 
it can be that the function is not located in a global minimum. In the present work, when this exit flag is present, a complete sweep on a wide range of possible initial guesses is conducted to ensure that the solution is located in a global minimum.

The systematic convergence of the optimization algorithm to a point which is not a root, when the initial guess is not close enough to the global solution, demonstrates that the objective function has local minima with values near to the global minimum. To illustrate this behavior, Fig. 21 shows, in a logarithmic scale, the absolute, real and imaginary values of the objective function, as a function of the optimization algorithm's independent variable, the azimuthal wave number $k_{z}$, for the frequency of $1000 \mathrm{~Hz}$. Analyzing the
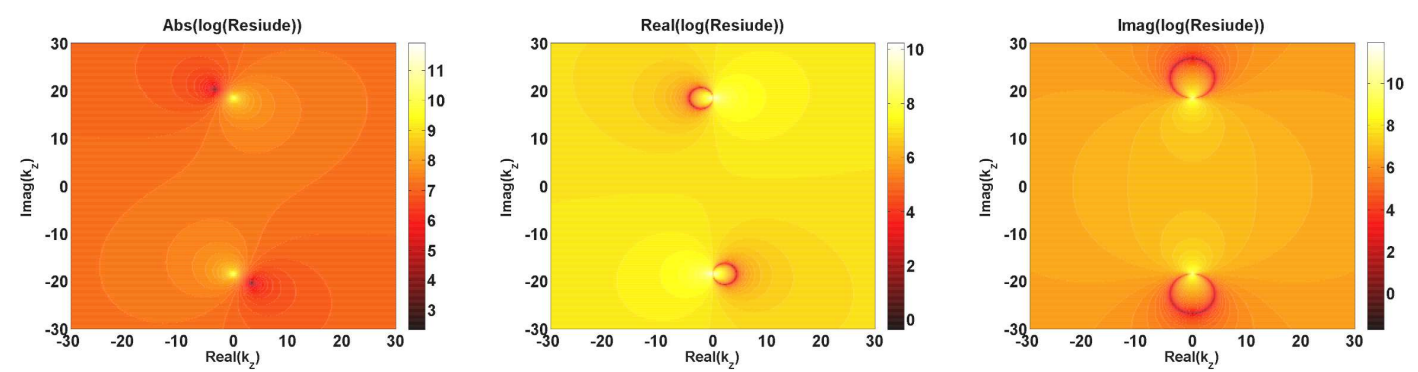

Figure 21: Absolute, real and imaginary values of the objective function, as a function of the azimuthal wave number $k_{z}$. (Sample $2, \mathrm{f}=1000 \mathrm{~Hz}, \mathrm{M}=0.0$ )

absolute value of the objective function, Fig. 21, it is seen - a priori - that the objective function has two major candidate regions to locate the global minimum. However, as the causality condition requires that the resistance of an acoustic liner must be positive $\left(\operatorname{Re}\left(Z_{w x}\right)>0\right)$, only the solution with $\operatorname{Re}\left(k_{z}\right)>0$ will result in a physical impedance value. The optimization algorithm minimizes simultaneously the real and imaginary components of the residue. For this reason both quantities are analyzed individually. For both components, a region with ring format is noticeable, which characterizes a local minimum region. A detailed view of the region of interest is presented in Fig. 22. Figure 22 shows very narrow spots which may locate a global minimum. For the real component, at least 10 spots candidates to a global minimum, while, for the imaginary component figure, the residual function presents at least 11 possible spots, with $R e\left(k_{z}\right)>0$. It is seen, in addition, that the objective function residual values, on the global minimum candidate 

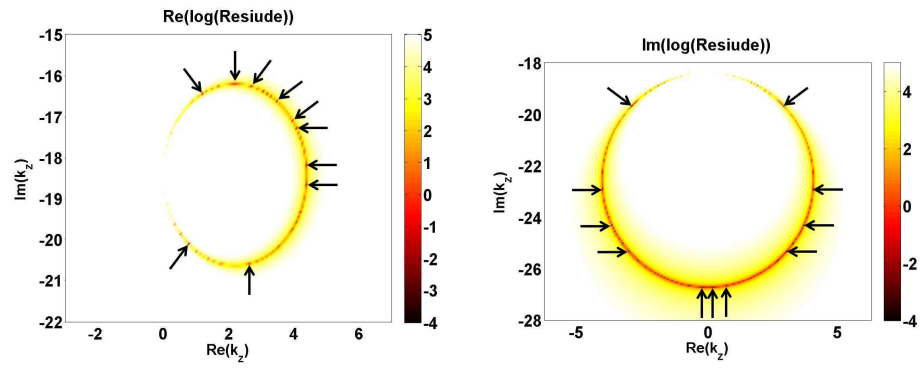

Figure 22: Detailed view of the real and imaginary values of the objective function, as a function of the azimuthal wave number $k_{z}$. (Sample 2, $\mathrm{f}=1000 \mathrm{~Hz}, \mathrm{M}=0.0$ )

points, is higher on the real component than on the imaginary ones.

The very narrow objective function minima characteristics, on candidate regions to locate the global minimums, and the residue real and imaginary scales disparity bring important challenges to the adopted optimization routine.

\section{Conclusions}

In this paper, an analytical technique for the indirect acoustic impedance eduction in presence of grazing flows is presented, numerically verified and applied to two acoustic liner configurations which are representative for airplane nacelles applications. The experimental results are compared with impedance values that are obtained on a different test rig using a different eduction methodology, and show a good agreement. A sensitivity analysis shows the effects, on the educed impedance curve, of inaccuracies on the calculation of the input parameters. This sensitivity analysis also shows the importance of taking into account the role of the rigid-/impedance wall transition, for an accurate impedance eduction. Considerations about the non-linear optimization algorithm demonstrates that it can converge to a local minimum and can results in incorrect impedance estimations.

It is shown that the proposed technique is capable to detect deviations on the impedance curve caused by non-linear interactions between the fluid dynamic and the acoustic field on the liner surface. The benefits of the approach include predominantly the sample non-destructivity, straight-forward rig construction, operation and a reduced number of microphones, since the acoustic plane wave propagation hypothesis on either side of the two-port 
system is respected. With the means discussed in this paper, the methodology can be successfully adopted for engineering applications.

\section{Acknowledgments}

Leandro D. Santana acknowledges the financial support of the European Commission through the Marie-Curie Research and Training Network "AETHER", Contract Nr. MRTN-CT-2006-035713 and the Brazilian Coordination for Improvement of Higher Education Personnel - CAPES - process number BEX-0520-10-1. The authors acknowledge Alenia Aermacchi S.p.A. for the supply of the samples analyzed in this paper.

[1] P. Dean, An in situ method of wall acoustic impedance measurement in flow ducts, Journal of Sound and Vibration 34 (1) (1974) $97-130$. doi:10.1016/S0022-460X(74)80357-3.

[2] T. Elnady, H. Bodén, An inverse analytical method for extracting liner impedance from pressure measurements, in: $10^{\text {th }}$ AIAA/CEAS Aeroacoustics Conference, no. AIAA 2004-2836, 2004, pp. 434-450. doi:10.2514/6.2004-2836.

[3] W. R. Watson, A new method for determining acoustic-liner admittance in a rectangular duct with grazing flow from experimental data, Tech. rep., National Aeronautics and Space Administration. Langley Research Center (1984).

[4] W. R. Watson, A method for determining acoustic-liner admittance in ducts with sheared flow in two-cross-sectional directions, Tech. rep., National Aeronautics and Space Administration. Langley Research Center (1985).

[5] W. R. Watson, M. B. T. M. G. Jones, T. L. Parrott, Impedance eduction in the presence of shear flow, in: $7^{\text {th }}$ AIAA/CEAS Aeroacoustics Conference, 2001. doi:10.2514/6.2001-2263.

[6] A. Scofano, P. B. Murray, P. Ferrante, Back-calculation of liner impedance using duct insertion loss measurements and fem predictions, in: $13^{\text {th }}$ AIAA/CEAS Aeroacoustics Conference (28th AIAA Aeroacoustics Conference), 2007. doi:10.2514/6.2007-3534. 
[7] W. R. Watson, M. G. Jones, Impedance eduction in ducts with higherorder modes and flow, in: $15^{t h}$ AIAA/CEAS Aeroacoustics Conference (30th AIAA Aeroacoustics Conference), no. AIAA 2009-3236, 2009. doi:10.2514/6.2009-3236.

[8] Y. Aurégan, M. Leroux, V. Pagneux, Measurement of liner impedance with flow by an inverse method, in: $10^{\text {th }}$ AIAA/CEAS Aeroacoustics Conference, no. AIAA 2004-2838, 2004. doi:10.2514/6.2004-2838.

[9] T. Elnady, M. Musharrof, H. Bodén, B. Elhadidi, Validation of an inverse analytical technique to educe liner impedance with grazing flow, in: $12^{\text {th }}$ AIAA/CEAS Aeroacoustics Conference (27th AIAA Aeroacoustics Conference), no. AIAA 2006-2639, 2006. doi:10.2514/1.41647.

[10] G. Gabard, Mode-matching techniques for sound propagation in lined ducts with flow, in: $16^{\text {th }}$ AIAA/CEAS Aeroacoustics Conference, no. AIAA 2010-3940, 2010. doi:10.2514/6.2010-3940.

[11] S. Busse, C. Richter, F. H. Thiele, C. Heuwinkel, L. Enghardt, I. Röhle, U. Michel, P. Ferrante, A. Scofano, Impedance deduction based on insertion loss measurements of liners under grazing flow conditions, in: $14^{\text {th }}$ AIAA/CEAS Aeroacoustics Conference (29th AIAA Aeroacoustics Conference), no. AIAA 2008-3014, 2008. doi:10.2514/6.2008-3014.

[12] W. De Roeck, W. Desmet, Indirect acoustic impedance determination in flow ducts using a two-port formulation, in: $15^{\text {th }}$ AIAA/CEAS Aeroacoustics Conference (30th AIAA Aeroacoustics Conference), no. AIAA 2009-3302, 2009. doi:10.2514/6.2009-3302.

[13] H. Bodén, Polyharmonic distortion modelling applied to acoustic characterisation of peforates, in: $17^{\text {th }}$ AIAA/CEAS Aeroacoustics Conference (32 ${ }^{\text {nd }}$ AIAA Aeroacoustics Conference), no. AIAA 2011-2724, 2011. doi:10.2514/6.2011-2724.

[14] M. Ábom, Measurement of the scattering-matrix of acoustical twoports, Mechanical Systems and Signal Processing 5 (2) (1991) 89-104. doi:10.1016/0888-3270(91)90017-Y.

[15] W. De Roeck, Hybrid methodologies for the computational analysis of confined subsonic flows, Ph.D. thesis, Katholieke Universiteit Leuven (2007). 
[16] E. Dokumaci, On the propagation of plane sound waves in ducts carrying an incompressible axial mean flow having an arbitrary velocity profile, Journal of Sound and Vibration 249 (4) (2002) 824 - 827. doi:10.1006/jsvi.2001.3744.

[17] E. Dokumaci, A plane wave approximation to sound transmission in parallel sheared mean flow, Journal of Sound and Vibration 284 (2005) 551565. doi:10.1016/j.jsv.2004.06.037.

[18] M. L. Munjal, A. G. Doige, Theory of a two source-location method for direct experimental evaluation of the four-pole parameters of an aeroacoustic element, Journal of Sound and Vibration 141 (2) (1990) 323 333. doi:10.1016/0022-460X(90)90843-O.

[19] T. Y. Lung, A. G. Doige, A time-averaging transient testing method for acoustic properties of piping systems and mufflers with flow, The Journal of the Acoustical Society of America 73 (3) (1983) 867-876. doi:10.1121/1.389056.

[20] U. Ingard, Influence of fluid motion past a plane boundary on sound reflection, absorption, and transmission, The Journal of the Acoustical Society of America 31 (7) (1959) 1035-1036. doi:10.1121/1.1907805.

[21] M. Myers, On the acoustic boundary condition in the presence of flow, Journal of Sound and Vibration 71 (3) (1980) 429 - 434. doi:10.1016/0022-460X(80)90424-1.

[22] S. W. Rienstra, Impedance models in time domain including the extended helmholtz resonator model, in: $12^{\text {th }}$ AIAA/CEAS Aeroacoustics Conference, 2006. doi:10.2514/6.2006-2686.

[23] W. De Roeck, W. Desmet, Experimental acoustic identification of flow noise sources in expansion chambers, in: Proceedings of ISMA 2008, 2008, pp. $455-470$.

[24] M. Åbom, H. Bodén, Error analysis of two microphone measurements in ducts with flow, The Journal of the Acoustical Society of America 83 (6) (1988) 2429-2438. doi:http://dx.doi.org/10.1121/1.396322.

[25] D. Marx, Y. Aurégan, H. Bailliet, J. Valière, PIV and LDV evidence of hydrodynamic instability over a liner ina duct with flow, 
Journal of Sound and Vibration 329 (18) (2010) 3798 - 3812. doi:10.1016/j.jsv.2010.03.025.

[26] A. Holmberg, M. Åbom, H. Bodén, Accurate experimental two-port analysis of flow generated sound, Journal of Sound and Vibration 330 (26) (2011) 6336 - 6354. doi:10.1016/j.jsv.2011.07.041.

[27] R. Boonen, P. Sas, W. Desmet, W. Lauriks, G. Vermeir, Calibration of the two microphone transfer function method with hard wall impedance measurements at different reference sections, Mechanical Systems and Signal Processing 23 (5) (2009) 1662-1671. doi:10.1016/j.ymssp.2008.12.001.

[28] L. D. Santana, W. De Roeck, W. Desmet, P. Ferrante, Two-port indirect acoustic impedance eduction in presence of grazing flows, in: $17^{\text {th }}$ AIAA/CEAS Aeroacoustics Conference (32 ${ }^{\text {nd }}$ AIAA Aeroacoustics Conference), no. AIAA 2011-2868, 2011. doi:10.2514/6.2011-2868. 